\title{
Factors Associated with Worsening Oxygenation in Patients with Non-severe COVID-19 Pneumonia
}

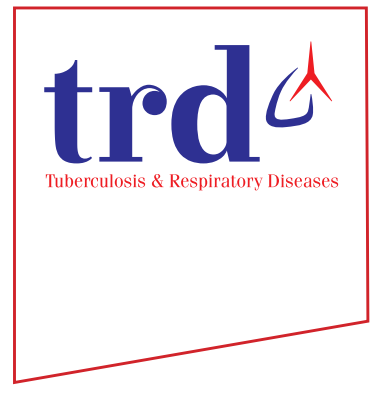

\author{
Cho Rom Hahm, M.D. ${ }^{1}{ }^{(\mathbb{D})}$, Young Kyung Lee, M.D., Ph.D. ${ }^{2}$, Dong Hyun Oh, M.D. ${ }^{1}$, Mi Young Ahn, \\ M.D., Ph.D. ', Jae-Phil Choi, M.D. ${ }^{1}$, Na Ree Kang, M.D. ${ }^{1}$, Jungkyun Oh, M.D. ${ }^{3}$, Hanzo Choi, M.D., \\ M.S. ${ }^{4}$ and Suhyun Kim, M.D. ${ }^{1}$ (i) \\ Departments of ${ }^{1}$ Internal Medicine, ${ }^{2}$ Radiology, and ${ }^{3}$ Hospital Medicine, Seoul Medical Center, Seoul, ${ }^{4}$ Department of \\ Emergency Medicine, Myong Ji St. Mary's Hospital, Seoul, Republic of Korea
}

Background: This study aimed to determine the parameters for worsening oxygenation in non-severe coronavirus disease 2019 (COVID-19) pneumonia.

Methods: This retrospective cohort study included cases of confirmed COVID-19 pneumonia in a public hospital in South Korea. The worsening oxygenation group was defined as that with $\mathrm{SpO}_{2} \leq 94 \%$ or received oxygen or mechanical ventilation (MV) throughout the clinical course versus the non-worsening oxygenation group that did not experience any respiratory event. Parameters were compared, and the extent of viral pneumonia from an initial chest computed tomography (CT) was calculated using artificial intelligence (AI) and measured visually by a radiologist.

Results: We included 136 patients, with $32(23.5 \%)$ patients in the worsening oxygenation group; of whom, two needed MV and one died. Initial vital signs and duration of symptoms showed no difference between the two groups; however, univariate logistic regression analysis revealed that a variety of parameters on admission were associated with an increased risk of a desaturation event. A subset of patients was studied to eliminate potential bias, that ferritin $\geq 280 \mu \mathrm{g} / \mathrm{L}$ ( $\mathrm{p}=0.029)$, lactate dehydrogenase $\geq 240 \mathrm{U} / \mathrm{L}(\mathrm{p}=0.029)$, pneumonia volume $(\mathrm{p}=0.021)$, and extent $(\mathrm{p}=0.030)$ by $\mathrm{AI}$, and visual severity scores $(\mathrm{p}=0.042)$ were the predictive parameters for worsening oxygenation in a sex-, age-, and comorbid illness-matched case-control study using propensity score $(\mathrm{n}=52)$.

Conclusion: Our study suggests that initial CT evaluated by AI or visual severity scoring as well as serum markers of inflammation on admission are significantly associated with worsening oxygenation in this COVID-19 pneumonia cohort.

Keywords: Pneumonia; COVID-19; Computed Tomography; Artificial Intelligence; Oxygenation

Address for correspondence: Suhyun Kim, M.D.

Department of Internal Medicine, Seoul Medical Center, 156 Shinnae-ro, Jungnang-gu, Seoul 02053, Republic of Korea

Phone: 82-2-2276-7814, Fax: 82-2-2276-7820

E-mail: sammy7597@naver.com

Received: Nov. 14, 2020

Revised: Dec. 29, 2020

Accepted: Jan. 1, 2021

Published online: Jan. 5, 2021

(c) It is identical to the Creative Commons Attribution Non-Commercial License (http://creativecommons.org/licenses/by-nc/4.0/).

Copyright $\odot 2021$

The Korean Academy of Tuberculosis and Respiratory Diseases.

\section{Introduction}

Coronavirus disease 2019 (COVID-19) has now become a pandemic since the first case of severe acute respiratory syndrome coronavirus 2 (SARS-CoV-2) in Wuhan, China ${ }^{1}$. The symptoms vary widely, from asymptomatic disease to pneumonia, to life-threatening complications and ultimately death. Sometimes it shows rapid progression of respiratory failure soon after the onset of dyspnoea and hypoxemia ${ }^{2}$. It is important to unravel the risk factors associated with hypoxemia and identify patients who are susceptible to severe or critical illness upon infection.

Older patients and those with pre-existing respiratory or 
cardiovascular conditions, diabetes, hypertension, and cancer appear to be at the greatest risk for poor outcomes or severe complications ${ }^{1,3-5}$. Leucocytosis, low lymphocyte count, serum levels of D-dimer, lactate dehydrogenase (LDH), ferritin, cardiac troponin, and higher concentrations of proinflammatory cytokines were reported to be risk factors associated with severe and fatal COVID-19 in hospitalized patients ${ }^{1,6,7}$. Typical imaging features of COVID-19 were established to help early screening and evaluation of the severity and extent of disease. Computed tomography (CT) involvement scores can assist in evaluation of the severity and extent of the disease ${ }^{8}$. Previous studies estimating risk factors included mostly people with severe and fatal diseases at presentation, and data are lacking for subclinical or non-severe COVID-19 patients. We explored potential risk factors associated with worsening oxygenation in a retrospective cohort of patients with non-severe COVID-19 pneumonia.

\section{Materials and Methods}

\section{Study design and participants}

This retrospective, single-center study, recruited hospitalized patients from 30 January to 15 April, 2020 in a public teaching hospital that also served as a COVID-19 dedicated hospital in Seoul, South Korea. We reviewed patients with COVID-19 pneumonia who routinely underwent non-contrast CTs at admission according to the hospital protocol. Ethical approval was provided by the institutional ethics review board of our hospital (2020-04-024). The requirement for informed consent was waived because of the retrospective study design. All those who tested positive SARS-CoV-2 in Korea were either hospitalized or monitored in community isolation settings, and discharged after twice consecutive SARS-CoV2 tests converted to negative. The inclusion criteria were patients aged $\geq 18$ years who (1) were positive for nucleic acid detection of SARS-CoV-2, (2) underwent a chest CT within 24 hours of admission, and (3) showed evidence of viral pneumonia in the initial chest CT scan. The presence of ground-glass opacity (GGO) or consolidation in the bilateral peripheral posterior lungs on chest CT scans without any other specified causes or pathogen in a COVID-19 confirmed patient was categorized as CT-documented COVID-19 pneumonia ${ }^{9}$. The exclusion criteria were (1) patients with symptom onset more than 14 days; (2) normal findings on initial chest CT; and (3) lung abnormalities other than viral pneumonia.

The clinician's decision dictated treatment. Multidisciplinary teams screened for signs of acute deterioration and the need for intensive care unit (ICU) admission daily using an in-hospital electronic-medical-record monitoring system.

\section{Data collection and definitions}

Demographic characteristics of the patients, laboratory and radiological data were reviewed. The need for oxygen devices such as nasal oxygen supply, high-flow oxygen therapy, or respiratory support such as invasive mechanical ventilation (MV) were assessed.

For classification of the clinical course with poor oxygenation throughout the hospitalization, we grouped patients into the worsening oxygenation group and the non-worsening group. The worsening oxygenation group was defined as having any of the following criteria for admission or transfer to the ICU: (1) desaturation event with peripheral oxygen saturation of hemoglobin $\left(\mathrm{SpO}_{2}\right)$ on room air with rest $\leq 94 \%$ in at least two consecutive measurements or (2) respiratory failure requiring high-flow oxygen therapy or MV. The non-worsening group referred to those who never desaturated and did not need supplemental oxygen.

According to the 'Diagnosis and Treatment Program of Pneumonia of COVID-19,' severe cases were defined as having any of the following criteria: (1) respiratory rate $\geq 30$ breaths/min; (2) resting blood oxygen saturation $\leq 93 \%$; or (3) partial pressure of arterial blood oxygen/fraction of inspired oxygen $\leq 300 \mathrm{~mm} \mathrm{Hg}$. Critical patients needed to meet one of the following conditions: (1) respiratory failure requiring MV, (2) shock, or (3) other organ failure requiring ICU monitoring or treatment ${ }^{10}$. We defined "non-severe COVID-19 patients" as those who did not meet any of the severe or critical criteria on admission.

\section{Chest CT acquisition}

Chest CTs were performed using a 64-detector row CT scanner (SOMATOM Sensation 64, Siemens Healthcare, Erlangen, Germany) on admission (low-dose [reference mAs, 40] with automatic exposure control; slice thickness, $1.0 \mathrm{~mm}$; and reconstruction interval, $3.0 \mathrm{~mm}$ ). Axial images were reconstructed with a sharp reconstruction kernel at a $3 \mathrm{~mm}$ slice thickness. The CTs were performed with the patient in the supine position at full inspiration without contrast medium. Axial images were reconstructed with a sharp reconstruction kernel at a $3 \mathrm{~mm}$ slice thickness.

\section{Quantitative CT analysis}

CT images of each patient were uploaded in a free software for artificial intelligence (AI)-driven automatic CT analysis of COVID-19 pneumonia (MEDIP v1.2.0.0, MEDICALIP Co. Ltd., Seoul, Korea), released on a website (http://medicalip. com/mobile/shop/covid19.php) on March 18, 2020. The deep learning software automatically generated a volumetric mask of the lung and pneumonia. The CT parameters from the automatic quantitative software were: the total lung volume 
$\left(\mathrm{cm}^{3}\right)$, the pneumonia volume $\left(\mathrm{cm}^{3}\right)$, pneumonia extent, and the mean lung densities of the total lung and the pneumonia.

An experienced thoracic radiologist (Y.K.L), blinded to the clinical data and laboratory indicators, evaluated the visual severity scores of the CT images. The CT severity score is adapted from a method previously used in patients after severe acute respiratory syndrome ${ }^{11}$. The extent of lung lesions was scored from 0 to 5 in each lobe and summed up (0, normal; 1, 1\%-5\% involvement; 2, 6\%-25\% involvement; 3, 26\%-50\% involvement; 4, 51\%-75\%; 5, 76\%-100\% involvement). The CT severity score was defined as the sum of the individual scores in the five lobes, which ranged from 0 to 25 points.

\section{Statistical analysis}

Continuous variables were represented as mean and standard deviation or median and interquartile ranges (IQR). A one-sample Kolmogorov-Smirnov test was performed to check the normality of all variables. To compare the two groups, the Mann-Whitney U test was used for continuous variables, and the chi-square or Fisher exact test was used for categorical variables. Correlation analysis was performed to identify associations between continuous variables. Univariate logistic regression analysis was performed to identify the clinical variables associated with worsening oxygenation events. Finally, a propensity score matching analysis was performed to minimize the effect of potential confounding factors that could affect the worsening oxygenation of COVID-19 pneumonia patients. The propensity score was estimated using a non-parsimonious logistic regression, with worsening oxygenation event as the dependent variable and all the characteristics as covariates (sex-, age-, and underlying-disease) based on their methodological, statistical, and clinical relevance. The patients with worsening oxygenation were matched 1:1 with the patients without worsening event according to the propensity score using exact matching. To assess bias reduction using the propensity score matching method, absolute standardized differences were calculated, with a value of $>20 \%$ indicating a meaningful imbalance in the baseline covariate. We selected 26 matched patients from each group to perform a case-control study at a ratio of 1:1, and a univariate logistic regression analysis was also performed with matched data. All statistical analyses were performed using SPSS statistical software version 23.0 (IBM Corp., Armonk, NY, USA) and R software version 3.6.2 (R Foundation for Statistical Computing, Vienna, Austria). p-values were based on a two-sided significance level of 0.05 .

\section{Results}

\section{Demographics and clinical courses of enrolled patients}

A total of 244 patients were admitted with laboratory-confirmed COVID-19 (Figure 1). After excluding 97 patients with normal baseline CT scans, four patients whose symptom onset was $>14$ days, and seven patients with bacterial coinfection or mycobacterial disease, 136 patients were included in this study (56 men, 80 women; mean age, $44.8 \pm 17.4$ years; range, 18-95 years). No patient received MV or high-flow oxygen therapy on admission, only one patient had $\mathrm{SpO}_{2}$ on room air $\leq 93 \%$, and two patients showed respiratory rate $\geq 30$ breaths/ min, there was no shock or organ failure at presentation. Most patients, except for three patients, were classified as having non-severe COVID-19 on admission ${ }^{10}$.

Among the 136 patients, 32 patients (23.5\%) were classified into the worsening oxygenation group, and 104 remained in the non-worsening group (76.5\%). All patients in the worsening oxygenation group had oxygen desaturation $\leq 94 \%$ in at least two consecutive measurements, and 10 patients required oxygen supplementation. Only two patients in the worsening oxygenation group needed MV, while a total of five patients needed high-flow oxygen therapy before or without MV.

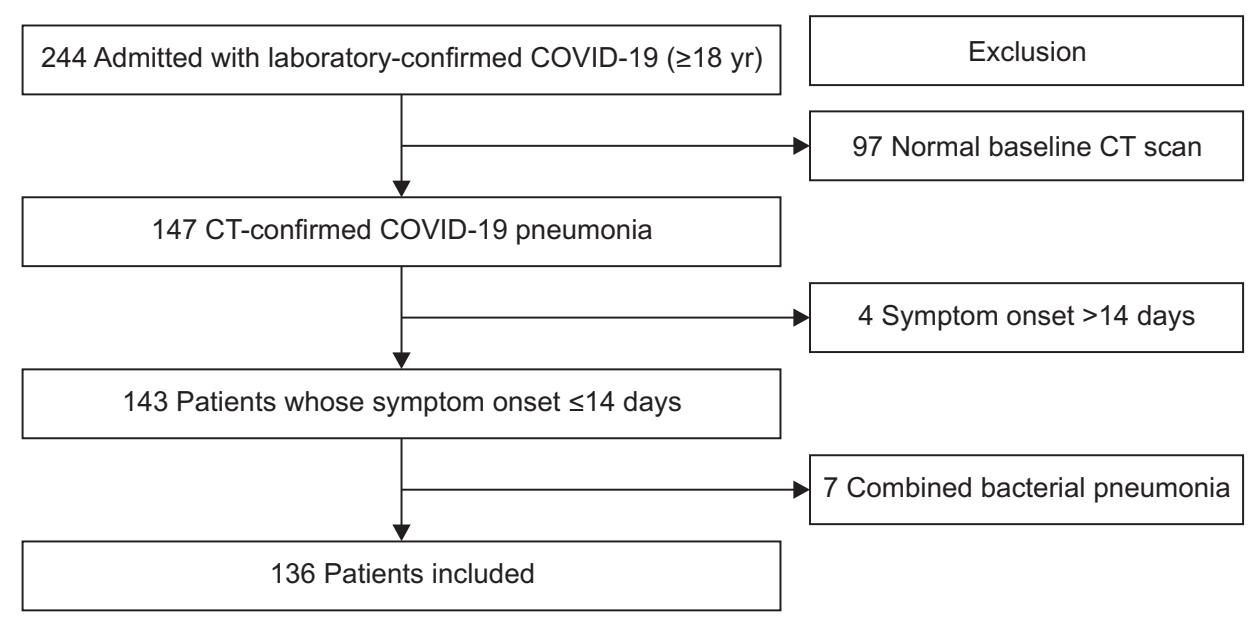

Figure 1. Study flow diagram. COVID-19: coronavirus disease 19; CT: computed tomography. 
Table 1. Baseline characteristics of hospitalized COVID-19 pneumonia patients

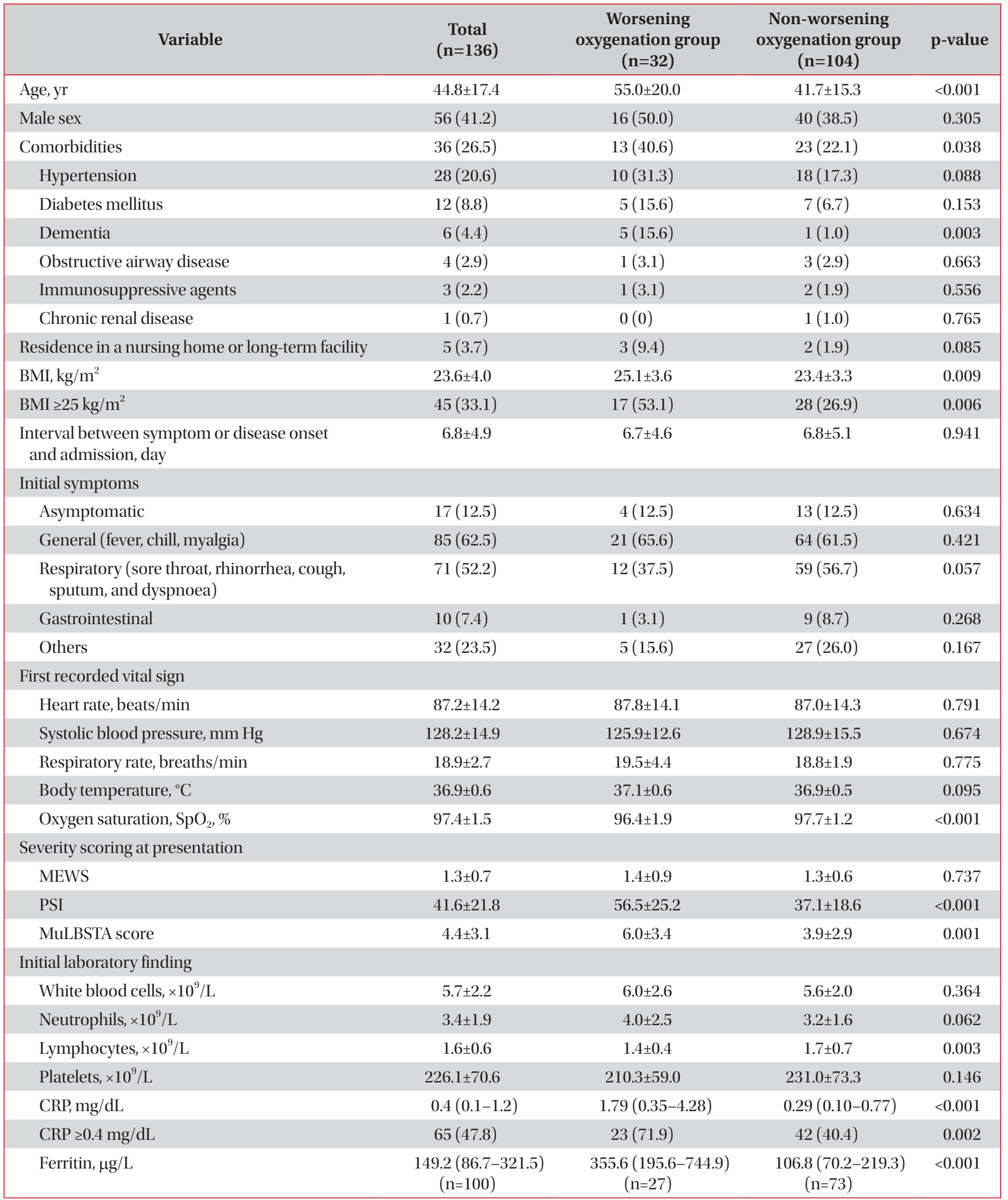


Table 1. Continued

\begin{tabular}{|c|c|c|c|c|}
\hline Variable & $\begin{array}{c}\text { Total } \\
(n=136)\end{array}$ & $\begin{array}{c}\text { Worsening } \\
\text { oxygenation group } \\
(n=32)\end{array}$ & $\begin{array}{l}\text { Non-worsening } \\
\text { oxygenation group } \\
(n=104)\end{array}$ & p-value \\
\hline Ferritin $\geq 280 \mu \mathrm{g} / \mathrm{L}$ & $29(29.0)$ & $16(50.0)$ & $13(12.5)$ & $<0.001$ \\
\hline LDH, U/L & $\begin{array}{c}245(213-309) \\
(\mathrm{n}=135)\end{array}$ & $\begin{array}{c}305(226-340) \\
(\mathrm{n}=32)\end{array}$ & $\begin{array}{c}236(212-289) \\
(\mathrm{n}=103)\end{array}$ & 0.006 \\
\hline $\mathrm{LDH} \geq 240 \mathrm{U} / \mathrm{L}$ & $51(37.8)$ & $19(59.4)$ & $32(30.8)$ & 0.003 \\
\hline
\end{tabular}

Values are presented as mean \pm SD, number (\%), or median (IQR).

$\mathrm{p}<0.05$ was considered statistically significant.

COVID-19: coronavirus disease 19; BMI: body mass index; MEWS: modified early warning score; PSI: pneumonia severity index; MuLBSTA score: multilobe infiltration, hypo-lymphocytosis, bacterial coinfection, smoking history, hypertension, and age; CRP: C-reactive protein; LDH: lactate dehydrogenase; SD: standard deviation; IQR: interquartile range.

Table 2. Comparison of parameters and scores of the initial low-dose chest CT scan between the two groups

\begin{tabular}{|c|c|c|c|c|}
\hline Variable & $\begin{array}{c}\text { Total } \\
(n=136)\end{array}$ & $\begin{array}{c}\text { Worsening } \\
\text { oxygenation group } \\
(n=32)\end{array}$ & $\begin{array}{c}\text { Non-worsening } \\
\text { oxygenation group } \\
(n=104)\end{array}$ & p-value \\
\hline Pneumonia volume, $\mathrm{cm}^{3}$ & $13.8(3.8-46.7)$ & $45.2(8.5-170.6)$ & $10.7(3.5-38.2)$ & 0.001 \\
\hline Extent of opacities, \% & $0.7(0.2-2.2)$ & $2.4(0.5-6.5)$ & $0.5(0.2-1.7)$ & 0.001 \\
\hline $0-5$ & $90(66.2)$ & $16(50.0)$ & $74(71.2)$ & \\
\hline $6-10$ & $29(21.3)$ & $4(12.5)$ & $25(24.0)$ & \\
\hline$\geq 11$ & $17(12.5)$ & $12(37.5)$ & $5(4.8)$ & \\
\hline
\end{tabular}

Values are presented as median (IQR), mean \pm SD, or number $(\%)$.

$\mathrm{p}<0.05$ was considered statistically significant.

CT: computed tomography; AI: artificial intelligence; IQR: interquartile range; SD: standard deviation.

No patient needed extracorporeal membrane oxygenation (ECMO). During hospitalization, 10 patients were admitted to the ICU, including two patients who developed acute respiratory distress syndrome, and one patient died declining to apply the MV in accordance with a previously signed advance directive. Median hospitalized days were $31.4 \pm 1$.1 without difference among two groups. Among the patients in the worsening oxygenation group, time intervals for oxygen desaturation $\leq 94 \%$ were a median 2 days (IQR, 1-5) from admission and 8 days (IQR, 6-11) from symptom or disease onset, respectively. The oxygen support was started after a median 3 days (IQR, 1-7) from admission and 12 days (IQR 9-13) from symptom or disease onset.

\section{Baseline characteristics and laboratory indices between the two groups}

The baseline characteristics of COVID-19 patients according to the clinical course are summarized in Table 1 . The aver- age interval for admission from symptom or disease onset was 6.8 days. Only 71 of 136 patients (52.2\%) complained of respiratory symptoms, and only 23 of 136 patients (16.9\%) had a body temperature $(\mathrm{BT}) \geq 37.5^{\circ} \mathrm{C}$ on admission. Intervals between symptom or disease onset and admission, and initial symptoms showed no difference between the two groups. The first recorded vital signs were similar in the two groups.

Patients in the worsening oxygenation group were older $(\mathrm{p}<0.001)$ and had more underlying diseases than those in the non-worsening group ( $40.6 \%$ vs. $22.1 \%$, $\mathrm{p}=0.038$ ). Hypertension was the most common underlying disease in both groups, and dementia was more frequent $(15.6 \%$ vs. $1.0 \%$, $\mathrm{p}=0.003$ ) in the worsening oxygenation group than in the nonworsening group. The worsening oxygenation group showed higher body mass index (BMI) and $53.1 \%$ had obesity based on a BMI $\geq 25 \mathrm{~kg} / \mathrm{m}^{2}$ (vs. $26.9 \%$, $\mathrm{p}=0.006$ ).

There were numerous differences in laboratory findings between the two groups (Table 1), including higher serum levels of C-reactive protein (CRP), ferritin, and LDH as well as 
lower lymphocyte counts in the worsening oxygenation group than in the non-worsening group (all $\mathrm{p}<0.05$ ). The worsening oxygenation group had higher pneumonia severity index (PSI); (56.5 vs. 37.1, $\mathrm{p}<0.001$ ) and MuLBSTA score (6.0 vs. 3.9, $\mathrm{p}=0.001$ ) than the non-worsening group, the modified early warning score (MEWS) showed no difference (1.4 vs. 1.3, $\mathrm{p}=0.737)$.

A

\begin{tabular}{|l|c|c|c|c|c|}
\hline & Volume $\left(\mathrm{cm}^{3}\right)$ & Mean (HU) & $\begin{array}{c}\text { Standard } \\
\text { deviation }\end{array}$ & $\begin{array}{c}\text { Pneumonia } \\
\text { burden }(\mathrm{g})\end{array}$ & $\begin{array}{c}\% \text { extent } \\
(\mathrm{ACR})\end{array}$ \\
\hline Lung & $2,353.8$ & -647.1 & 246.1 & - & - \\
\hline Pneumonia & 550.5 & -348.0 & 193.3 & 382.3 & $23.4 \%(0.2)$ \\
\hline
\end{tabular}
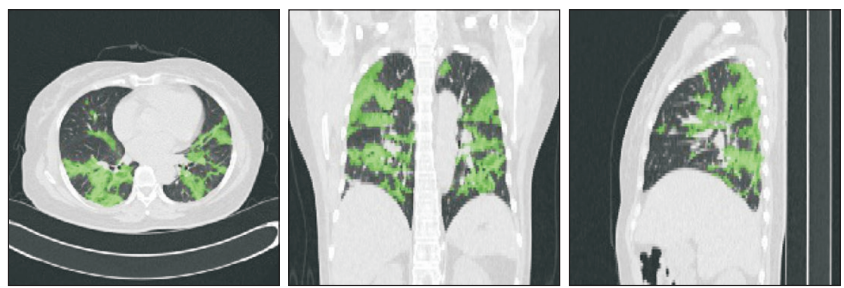

\section{Relationships between CT features and worsening oxygenation}

The pneumonia volume quantified using AI was significantly larger in the worsening oxygenation group (median, $45.2 \mathrm{~cm}^{3}$ vs. $10.7 \mathrm{~cm}^{3} ; \mathrm{p}=0.001$ ) as well as the extent of opacities (median, $2.4 \%$ vs. $0.5 \%$; $\mathrm{p}=0.001$ ). Visual severity scores of the CT images were also significantly higher in the worsening

B

\begin{tabular}{|l|c|c|c|c|c|}
\hline & Volume $\left(\mathrm{cm}^{3}\right)$ & Mean (HU) & $\begin{array}{c}\text { Standard } \\
\text { deviation }\end{array}$ & $\begin{array}{c}\text { Pneumonia } \\
\text { burden }(\mathrm{g})\end{array}$ & $\begin{array}{c}\% \text { extent } \\
(\mathrm{ACR})\end{array}$ \\
\hline Lung & $3,953.7$ & -819.9 & 140.0 & - & - \\
\hline Pneumonia & 34.3 & -462.2 & 180.1 & 19.6 & $0.9 \%(0.0)$ \\
\hline
\end{tabular}
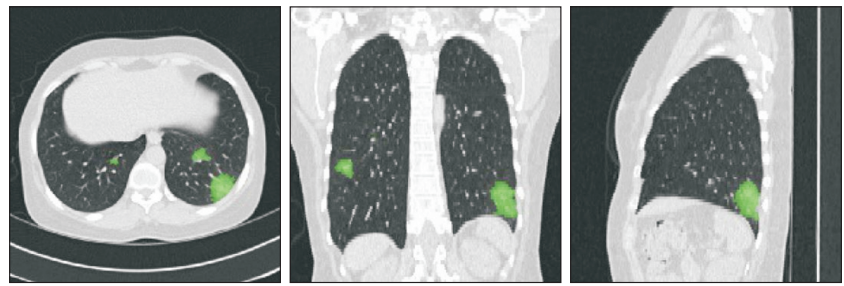

Figure 2. Coronavirus disease 2019 (COVID-19) pneumonia lesions detected by the artificial intelligence (AI) system and comparison of the interpretation of the quantification computed tomography parameters between the worsening oxygenation group (A) and the non-worsening oxygenation group (B). The area considered as COVID-19 pneumonia by AI is highlighted in green. The following images of two patients are illustrated. (A) One is a 55-year-old female who entered the worsening oxygenation group within 24 hours of admission. She presented with desaturation $\leq 94 \%$ within 24 hours of admission and worsened rapidly, requiring high-flow oxygen therapy on the 3rd day after admission. She was discharged on the $42 \mathrm{nd}$ day from admission. AI calculated a pneumonia volume of $382.3 \mathrm{~g}$ and pneumonia extent of $23.4 \%$. The radiologist scored 19/25 using the visual severity score. (B) The other image shows a 47-year-old female who did not meet the worsening oxygenation group criteria during follow-up and was discharged from the hospital after the 41st day without any respiratory event. AI calculated a pneumonia volume of $19.6 \mathrm{~g}$ and pneumonia extent of $0.9 \%$, and the radiologist scored $6 / 25$ using the visual severity score.

Table 3. Univariate analysis of predictive parameters for worsening oxygenation in patients with COVID-19 pneumonia $(\mathbf{n}=136)$

\begin{tabular}{|c|c|c|c|}
\hline Variable & OR & $95 \% \mathrm{CI}$ & p-value \\
\hline Age, yr & 1.047 & $1.021-1.074$ & $<0.001$ \\
\hline Dementia (vs. non-dementia) & 19.074 & $2.138-170.167$ & 0.008 \\
\hline BMI, $\mathrm{kg} / \mathrm{m}^{2}$ & 3.076 & $1.357-6.973$ & 0.007 \\
\hline PSI & 1.043 & $1.021-1.065$ & $<0.001$ \\
\hline MuLBSTA score & 1.269 & $1.097-1.469$ & 0.002 \\
\hline Lymphocyte count, $\times 10^{9} / \mathrm{L}$ & 0.320 & $0.137-0.750$ & 0.009 \\
\hline $\mathrm{CRP} \geq 0.4 \mathrm{mg} / \mathrm{dL}$ (vs. $<0.4 \mathrm{mg} / \mathrm{dL})$ & 3.772 & $1.589-8.955$ & 0.003 \\
\hline Ferritin $\geq 280 \mu \mathrm{g} / \mathrm{L}($ vs. $<280 \mu \mathrm{g} / \mathrm{L})$ & 7.000 & $2.834-17.293$ & $<0.001$ \\
\hline $\mathrm{LDH} \geq 240 \mathrm{U} / \mathrm{L}$ (vs. <240 U/L) & 3.288 & $1.450-7.460$ & 0.004 \\
\hline Pneumonia volume by AI, $\mathrm{cm}^{3}$ & 1.014 & $1.007-1.022$ & $<0.001$ \\
\hline Extent of opacities by AI, \% & 1.283 & $1.109-1.486$ & 0.001 \\
\hline Visual severity scoring of the CT by the radiologist & 1.225 & $1.105-1.359$ & $<0.001$ \\
\hline
\end{tabular}

$\mathrm{p}<0.05$ was considered statistically significant.

COVID-19: coronavirus disease 19; OR: odds ratio; CI: confidence interval; BMI: body mass index; PSI: pneumonia severity index; MuLBSTA score: multilobe infiltration, hypo-lymphocytosis, bacterial coinfection, smoking history, hypertension, and age; CRP: C-reactive protein; LDH: lactate dehydrogenase; AI: artificial intelligence; CT: computed tomography. 
oxygenation group ( $8.0 \pm 5.2$ vs. $4.6 \pm 3.2$, $p<0.001)$. In particular, $37.5 \%$ of the worsening oxygenation group showed a visual severity score $\geq 11 / 25$ (vs. $4.8 \%$ of the non-worsening group) (Table 2).

Figure 2 shows examples of COVID-19 pneumonia lesions from each group detected by free software for AI-driven automatic CT analysis.

\section{Predictors of worsening oxygenation}

We initially evaluated the results using univariate analysis
(Table 3). Our analysis revealed that elderly patients, dementia, obesity, higher pneumonia severity scores, such as the PSI and MuLBSTA score, higher levels of initial CRP, ferritin, and $\mathrm{LDH}$, and lower lymphocyte levels were associated with the worsening oxygenation group compared to the non-worsening group. In addition, pneumonia volume and the extent of the CT by AI, and visual severity scores, were higher in the worsening oxygenation group when compared with the nonworsening group $(\mathrm{p}<0.05)$.

After matching on the propensity score, 52 patients were included in the analysis, and between-group differences for

Table 4. Comparison of clinical parameters between the two groups in this matched case-control study ( $\mathrm{n}=52)$

\begin{tabular}{|c|c|c|c|}
\hline Variable & $\begin{array}{l}\text { Worsening oxygenation group } \\
(n=26)\end{array}$ & $\begin{array}{c}\text { Non-worsening oxygenation } \\
\text { group }(n=26)\end{array}$ & p-value \\
\hline Age, yr & $55.7 \pm 19.5$ & $50.5 \pm 12.5$ & 0.220 \\
\hline Male sex & $15(57.5)$ & $16(61.5)$ & 0.777 \\
\hline \multicolumn{4}{|l|}{ Comorbidities } \\
\hline Hypertension & $8(30.8)$ & $6(23.1)$ & 0.532 \\
\hline Diabetes mellitus & $4(15.4)$ & $3(11.5)$ & 0.999 \\
\hline $\mathrm{BMI}, \mathrm{kg} / \mathrm{m}^{2}$ & $24.9 \pm 3.6$ & $24.2 \pm 2.5$ & 0.207 \\
\hline \multicolumn{4}{|l|}{ Initial symptoms } \\
\hline Asymptomatic & $2(7.7)$ & $5(19.2)$ & 0.419 \\
\hline General (fever, chills, and myalgia) & $18(69.2)$ & $14(53.8)$ & 0.254 \\
\hline $\begin{array}{l}\text { Respiratory (sore throat, rhinorrhea, cough, } \\
\text { sputum, and dyspnea) }\end{array}$ & $11(42.3)$ & $13(50.0)$ & 0.578 \\
\hline Gastrointestinal & $1(3.8)$ & $2(7.7)$ & 0.999 \\
\hline Others & $5(19.2)$ & $5(19.2)$ & 0.999 \\
\hline \multicolumn{4}{|l|}{ First recorded vital signs } \\
\hline Heart rate, beats/min & $88.9 \pm 14.6$ & $90.5 \pm 14.2$ & 0.447 \\
\hline Systolic blood pressure, $\mathrm{mmHg}$ & $125.8 \pm 12.6$ & $132.3 \pm 16.1$ & 0.263 \\
\hline Respiratory rate, breaths/min & $19.1 \pm 3.9$ & $18.9 \pm 1.7$ & 0.728 \\
\hline Body temperature, ${ }^{\circ} \mathrm{C}$ & $37.1 \pm 0.5$ & $36.8 \pm 0.4$ & 0.094 \\
\hline Oxygen saturation, $\mathrm{SpO}_{2}, \%$ & $96.4 \pm 2.0$ & $96.9 \pm 1.2$ & 0.358 \\
\hline \multicolumn{4}{|l|}{ Severity scorings on presentation } \\
\hline MEWS & $1.35 \pm 0.8$ & $1.38 \pm 0.8$ & 0.827 \\
\hline PSI & $56.6 \pm 23.5$ & $48.4 \pm 16.5$ & 0.073 \\
\hline MuLBSTA score & $6.15 \pm 3.5$ & $4.9 \pm 2.8$ & 0.134 \\
\hline \multicolumn{4}{|l|}{ Initial complete blood count } \\
\hline White blood cells, $\times 10^{9} / \mathrm{L}$ & $5.6 \pm 1.9$ & $5.3 \pm 1.6$ & 0.379 \\
\hline Neutrophils, $\times 10^{9} / \mathrm{L}$ & $3.6 \pm 1.8$ & $3.1 \pm 1.2$ & 0.257 \\
\hline Lymphocytes, $\times 10^{9} / \mathrm{L}$ & $1.4 \pm 0.4$ & $1.6 \pm 0.6$ & 0.073 \\
\hline Platelets, $\times 10^{9} / \mathrm{L}$ & $205.9 \pm 62.5$ & $204.2 \pm 68.0$ & 0.971 \\
\hline
\end{tabular}

Values are presented as mean \pm SD or number $(\%)$.

$\mathrm{p}<0.05$ was considered statistically significant.

BMI: body mass index; MEWS: modified early warning score; PSI: pneumonia severity index; MuLBSTA score: multilobe infiltration, hypolymphocytosis, bacterial coinfection, smoking history, hypertensions and age. 
all covariates were eliminated (Table 4). After excluding the impact of age, underlying disease, initial symptoms and first recorded vital signs, initial pneumonia severity scoring, and complete blood count, ferritin $\geq 280 \mu \mathrm{g} / \mathrm{L}$ (odds ratio [OR], 3.600; 95\% confidence interval [CI], 1.142-11.346; $\mathrm{p}=0.029$ ), $\mathrm{LDH} \geq 240$ U/L (OR, 3.600; 95\% CI, 1.142-11.346; $\mathrm{p}=0.029$ ), pneumonia volume (OR, 1.010; 95\% CI, 1.002-1.019; $\mathrm{p}=0.021)$ and extent (OR, 1.194; 95\% CI, 1.017-1.401; $\mathrm{p}=0.030)$ by AI, and visual severity scores (OR, 1.146; 95\% CI, 1.005-1.307; $\mathrm{p}=0.042$ ) were the parameters for predicting worsening oxygenation in this cohort $(\mathrm{p}<0.05)$ (Table 5).

\section{Discussion}

We designed this study to identify parameters at an early stage to predict whether oxygen will decrease. Initial chest CT parameters by AI and visual severity scoring predicted worsening oxygenation in non-severe COVID-19 pneumonia even though initial vital signs at admission were not predictive for worsening oxygenation during hospitalization in our study. It is estimated that $15 \%-20 \%$ of COVID-19 patients develop severe pneumonia and $5 \%-10 \%$ require critical care ${ }^{12}$. Severe illness of COVID-19 usually begins approximately 1 week after the onset of symptoms ${ }^{2}$, while patients have variable phases of symptoms and disease onset at admission ${ }^{12}$. In the present study, most patients were classified as having nonsevere COVID-19 at presentation ${ }^{10}$, and no patient received MV or high-flow oxygen therapy on admission. Patients in this cohort were admitted an average of 6.8 days from symptom onset, and $12.5 \%$ were asymptomatic on admission. Nevertheless, $23.5 \%$ of these patients proceeded to oxygen desaturation during a median 2 days from hospitalization.

Early case series from the Korean Cohort Study showed that $21.4 \%$ of patients received oxygen therapy, with no requirement for $\mathrm{MV}^{13}$. In the Daegu and Gyeongbuk area in Korea, with substantial community outbreaks, a retrospective study (mean age of $55.4 \pm 17.1$ years) showed that $13.3 \%$ of patients required ICU admission with MV use in 11.2\%, ECMO in 4.1\%, and a mortality rate of $5.1 \%^{14}$. Studies on COVID-19 have been limited to patients with severe or critical disease admitted to the ICU, though a recent report showed that more than $80 \%$ of COVID-19 cases were mild in China ${ }^{4,6,15-20}$. In our study, most cases were non-severe, as evidenced by relatively low rates of ICU admission and mortality rates that would be related to younger ages (mean, 44.8 \pm 17.4 ) and lower comorbidity rates $(26.5 \%)$ than that of previous reports. In metropolitan Seoul, all confirmed cases were either hospitalized at dedicated hospitals or monitored at community isolation centers. Many cases of COVID-19 were diagnosed by surveillance testing in South Korea, so that a large proportion of the patients in our cohort had non-severe or subclinical symptoms at the beginning of hospitalization.

To our knowledge, markers for the prediction of worsening oxygenation among patients with initially non-severe COVID-19 pneumonia are not defined. In this cohort, only $16.9 \%$ showed fever based on a $\mathrm{BT} \geq 37.5^{\circ} \mathrm{C}$. Most of the first recorded vital signs were within normal ranges and showed no difference between the two groups, suggesting that the worsening oxygenation cannot be distinguished by initial vital signs. Apart from that, we noted that more patients in the worsening oxygenation group had higher pneumonia severity scores, and several laboratory parameters such as lymphopenia, higher ferritin, and LDH, as well as older age and comorbidities such as dementia and obesity were associated with a worsening oxygenation. Feng et al. ${ }^{10}$ suggested that the variance of the MuLBSTA score, may have a better predictive value in COVID-19 pneumonia as with this study ${ }^{21}$. Xie et al. ${ }^{22}$ suggested that traditional method such as the new early warning score may not help predict patients who will develop respiratory failure in COVID-19. Our data showed no difference in MEWS.

Our results also underline the importance of the integration of CT extent and severity into the management of COVID-19 pneumonia patients. Despite recent advances in molecular viral characterisation, recent evidence does not support a

Table 5. Univariate analysis of predictive parameters for worsening oxygenation in patients with COVID-19 pneumonia in this matched case-control study $(n=52)$

\begin{tabular}{|lccc|}
\hline \multicolumn{1}{|c}{ Variable } & OR & $\mathbf{9 5 \%}$ CI & p-value \\
\hline $\mathrm{CRP} \geq 0.4 \mathrm{mg} / \mathrm{dL}(\mathrm{vs} .<0.4 \mathrm{mg} / \mathrm{dL})$ & 2.224 & $0.627-7.890$ & 0.216 \\
\hline Ferritin $\geq 280 \mu \mathrm{g} / \mathrm{L}(\mathrm{vs} .<280 \mu \mathrm{g} / \mathrm{L})$ & 3.600 & $1.142-11.346$ & 0.029 \\
\hline $\mathrm{LDH} \geq 240 \mathrm{U} / \mathrm{L}(\mathrm{vs} .<240 \mathrm{U} / \mathrm{L})$ & 3.600 & $1.142-11.346$ & 0.029 \\
\hline Pneumonia volume by AI, cm ${ }^{3}$ & 1.010 & $1.002-1.019$ & 0.021 \\
\hline Extent of opacities by AI, $\%$ & 1.194 & $1.017-1.401$ & 0.030 \\
\hline Visual severity scoring of the CT by the radiologist & 1.146 & $1.005-1.307$ & 0.042 \\
\hline
\end{tabular}

$\mathrm{p}<0.05$ was considered statistically significant.

COVID-19: coronavirus disease 19; OR: odds ratio; CI: confidence interval; CRP: C-reactive protein; LDH: lactate dehydrogenase; AI: artificial intelligence; CT: computed tomography. 
severity or mortality stratification based on viral load dynam$\mathrm{ics}^{23}$. However, CT extent or CT features were recognized as important factors for predicting a worsening prognosis ${ }^{23-25}$. This is consistent with the results of the current study. The rapid development of AI has significantly improved automatic lung segmentation technology, making it possible to quantify the lesions automatically ${ }^{25}$. Quantitative analysis of CT using AI tools could provide an automatic and objective estimation of the disease burden, facilitating imaging interpretation during the pandemic ${ }^{23}$. This study aimed to investigate the capability of quantitative CT imaging features combined with traditional clinical biomarkers in predicting progression to worsening oxygenation in the early stages of COVID-19. We used free software and found that the AI-driven parameters of pneumonia volume and extent of the whole lung in the initial chest CT predicted the worsening oxygenation in non-severe COVID-19 pneumonia. Our study suggests the additional benefit of the visual severity scores summing the estimated extent of each lobe and the integration of clinical and laboratory parameters.

This study has limitations. First, it is a single-center retrospective study. In our hospital, the standard cut-off value for admission or transfer to the ICU and change or add-on of antiviral agents was $94 \%$ of the $\mathrm{SpO}_{2}$ on ambient air. These criteria should be regarded as empirical, as there is no robust supporting evidence. Second, fewer patients were severe or critical compared to other studies. The majority of the worsening oxygenation group recovered without oxygen or MV, or ECMO. These favorable outcomes may not reflect the full prognosis of patients with COVID-19 in other areas. We did not analyse the factors that could affect outcomes such as regional differences, medical resources, and treatment options we applied as in a clinical trial. In addition, it might be concerned about generalizability as the great majority of the described patients would not be hospitalized elsewhere and are unlikely to undergo CT imaging in routine clinical practice. Nevertheless, these data, including patients with mild symptoms in a relatively early stage, are thought to be useful in predicting parameters for clinical course and triaging patients in real practice. Third, we did not analyze the CT scans at the time of deterioration or follow-up, and the features or patterns with GGO or consolidation. Finally, we did not rule out other causes of the worsening oxygenation such as microthrombi, pulmonary thromboembolism or myocardial injury.

Our study presents initial CT evaluated by AI or visual severity scoring as well as serum markers of inflammation such as ferritin and LDH at admission are significantly associated with worsening oxygenation in non-severe COVID-19 pneumonia cohort.

\section{Authors' Contributions}

Conceptualization: Kim S, Lee YK. Methodology: Kim S, Hahm CR, Oh DH. Formal analysis: Kim S, Choi H. Data curation: Choi JP, Kang NR. Investigation: Ahn MY, Oh J. Writing original draft preparation: Hahm CR, Kim S. Writing - review and editing: Kim S, Lee YK, Hahm CR, Oh DH. Approval of final manuscript: all authors.

\section{Conflicts of Interest}

No potential conflict of interest relevant to this article was reported.

\section{Acknowledgments}

We thank the members of the Seoul Medical Centre Fight Against COVID-19 Team (SMC-FACT), all members of the department of emergency medicine, and other colleagues in the field. We would like to thank Editage (www.editage.co.kr) for English language editing.

\section{Funding}

No funding to declare.

\section{References}

1. Wu C, Chen X, Cai Y, Xia J, Zhou X, Xu S, et al. Risk factors associated with acute respiratory distress syndrome and death in patients with coronavirus disease 2019 pneumonia in $\mathrm{Wu}-$ han, China. JAMA Intern Med 2020;180:934-43.

2. Berlin DA, Gulick RM, Martinez FJ. Severe COVID-19. N Engl J Med 2020;383:2451-60.

3. Weiss P, Murdoch DR. Clinical course and mortality risk of severe COVID-19. Lancet 2020;395:1014-5.

4. Grasselli G, Zangrillo A, Zanella A, Antonelli M, Cabrini L, Castelli A, et al. Baseline characteristics and outcomes of 1591 patients infected with SARS-CoV-2 admitted to ICUs of the Lombardy region, Italy. JAMA 2020;323:1574-81.

5. Yang J, Zheng Y, Gou X, Pu K, Chen Z, Guo Q, et al. Prevalence of comorbidities and its effects in patients infected with SARS-CoV-2: a systematic review and meta-analysis. Int J Infect Dis 2020;94:91-5.

6. Yang X, Yu Y, Xu J, Shu H, Xia J, Liu H, et al. Clinical course and outcomes of critically ill patients with SARS-CoV-2 pneumonia in Wuhan, China: a single-centered, retrospective, observational study. Lancet Respir Med 2020;8:475-81.

7. Velavan TP, Meyer CG. Mild versus severe COVID-19: labora- 
tory markers. Int J Infect Dis 2020;95:304-7.

8. Zhao W, Zhong Z, Xie X, Yu Q, Liu J. Relation between chest CT findings and clinical conditions of coronavirus disease (COVID-19) pneumonia: a multicenter study. AJR Am J Roentgenol 2020;214:1072-7.

9. Pan F, Ye T, Sun P, Gui S, Liang B, Li L, et al. Time course of lung changes at chest CT during recovery from coronavirus disease 2019 (COVID-19). Radiology 2020;295:715-21.

10. Feng Y, Ling Y, Bai T, Xie Y, Huang J, Li J, et al. COVID-19 with different severities: a multicenter study of clinical features. Am J Respir Crit Care Med 2020;201:1380-8.

11. Chang YC, Yu CJ, Chang SC, Galvin JR, Liu HM, Hsiao CH, et al. Pulmonary sequelae in convalescent patients after severe acute respiratory syndrome: evaluation with thin-section CT. Radiology 2005;236:1067-75.

12. Guan WJ, Ni ZY, Hu Y, Liang WH, Ou CQ, He JX, et al. Clinical characteristics of coronavirus disease 2019 in China. N Engl J Med 2020;382:1708-20.

13. Kim ES, Chin BS, Kang CK, Kim NJ, Kang YM, Choi JP, et al. Clinical course and outcomes of patients with severe acute respiratory syndrome coronavirus 2 infection: a preliminary report of the first 28 patients from the Korean cohort study on COVID-19. J Korean Med Sci 2020;35:e142.

14. Hong KS, Lee KH, Chung JH, Shin KC, Choi EY, Jin HJ, et al. Clinical features and outcomes of 98 patients hospitalized with SARS-CoV-2 infection in Daegu, South Korea: a brief descriptive study. Yonsei Med J 2020;61:431-7.

15. Wu Z, McGoogan JM. Characteristics of and important lessons from the coronavirus disease 2019 (COVID-19) outbreak in China: summary of a report of 72314 cases from the Chinese Center for Disease Control and Prevention. JAMA 2020;323:1239-42.

16. Wang D, Hu B, Hu C, Zhu F, Liu X, Zhang J, et al. Clinical
Characteristics of 138 Hospitalized patients with 2019 novel coronavirus-infected pneumonia in Wuhan, China. JAMA 2020;323:1061-9.

17. Huang C, Wang Y, Li X, Ren L, Zhao J, Hu Y, et al. Clinical features of patients infected with 2019 novel coronavirus in Wuhan, China. Lancet 2020;395:497-506.

18. Bhatraju PK, Ghassemieh BJ, Nichols M, Kim R, Jerome KR, Nalla AK, et al. COVID-19 in critically ill patients in the Seattle region: case series. N Engl J Med 2020;382:2012-22.

19. Zhou F, Yu T, Du R, Fan G, Liu Y, Liu Z, et al. Clinical course and risk factors for mortality of adult inpatients with COVID-19 in Wuhan, China: a retrospective cohort study. Lancet 2020;395:1054-62.

20. Chen J, Qi T, Liu L, Ling Y, Qian Z, Li T, et al. Clinical progression of patients with COVID-19 in Shanghai, China. J Infect 2020;80:e1-6.

21. Guo L, Wei D, Zhang X, Wu Y, Li Q, Zhou M, et al. Clinical features predicting mortality risk in patients with viral pneumonia: the MuLBSTA score. Front Microbiol 2019;10:2752.

22. Xie J, Tong Z, Guan X, Du B, Qiu H, Slutsky AS. Critical care crisis and some recommendations during the COVID-19 epidemic in China. Intensive Care Med 2020;46:837-40.

23. Huang L, Han R, Ai T, Yu P, Kang H, Tao Q, et al. Serial Quantitative chest CT assessment of COVID-19: deep-learning approach. Radiology Cardiothoracic Imaging 2020;2:e200075.

24. Luo Z, Wang N, Liu P, Guo Q, Ran L, Wang F, et al. Association between chest CT features and clinical course of Coronavirus Disease 2019. Respir Med 2020;168:105989.

25. Liu F, Zhang Q, Huang C, Shi C, Wang L, Shi N, et al. CT quantification of pneumonia lesions in early days predicts progression to severe illness in a cohort of COVID-19 patients. Theranostics 2020;10:5613-22. 